\title{
Dietary zinc supplementation of 3xTg-AD mice increases BDNF levels and prevents cognitive deficits as well as mitochondrial dysfunction
}

\author{
C Corona ${ }^{1,2}$, F Masciopinto ${ }^{1,2}$, E Silvestri ${ }^{3}$, A Del Viscovo ${ }^{3}$, R Lattanzio ${ }^{4}$, R La Sorda ${ }^{4}$, D Ciavardelli ${ }^{5}$, F Goglia ${ }^{3}$, M Piantelli ${ }^{4}$, \\ LMT Canzoniero ${ }^{3}$ and SL Sensi ${ }^{\star, 1,2,6}$
}

The overall effect of brain zinc $\left(\mathrm{Zn}^{2+}\right)$ in the progression and development of Alzheimer's disease (AD) is still not completely understood. Although an excess of $\mathrm{Zn}^{2+}$ can exacerbate the pathological features of $A D$, a deficit of $\mathrm{Zn}^{2+}$ intake has also been shown to increase the volume of amyloid plaques in $A D$ transgenic mice. In this study, we investigated the effect of dietary $\mathrm{Zn}^{2+}$ supplementation (30 p.p.m.) in a transgenic mouse model of $A D$, the 3xTg-AD, that expresses both $\beta$ amyloid $(A \beta)$ - and tau-dependent pathology. We found that $\mathrm{Zn}^{2+}$ supplementation greatly delays hippocampal-dependent memory deficits and strongly reduces both $A \beta$ and tau pathology in the hippocampus. We also evaluated signs of mitochondrial dysfunction and found that $\mathrm{Zn}^{2+}$ supplementation prevents the age-dependent respiratory deficits we observed in untreated 3XTg-AD mice. Finally, we found that $\mathrm{Zn}^{2+}$ supplementation greatly increases the levels of brain-derived neurotrophic factor (BDNF) of treated 3xTg-AD mice. In summary, our data support the idea that controlling the brain $\mathrm{Zn}^{2+}$ homeostasis may be beneficial in the treatment of AD. Cell Death and Disease (2010) 1, e91; doi:10.1038/cddis.2010.73; published online 28 October 2010

Subject Category: Neuroscience

Alzheimer's disease (AD) is the most common form of dementia and is characterized by the accumulation of amyloid- $\beta$ peptide $(\mathrm{A} \beta)$ and the formation of neurofibrillary tangles made of hyperphosphorylated tau (h-tau). The pathogenic role of metal dyshomeostasis in $A D$ has been largely debated in recent years. ${ }^{1} \mathrm{~A} \beta$ aggregation is, in fact, a process strongly potentiated by the peptide interaction with several metals and zinc $\left(\mathrm{Zn}^{2+}\right)$, in particular. ${ }^{2}$ In addition, at high concentrations $(250 \mu \mathrm{M}), \mathrm{Zn}^{2+}$ has been shown to trigger tau phosphorylation as well as its fibrillization., However, the effect of $\mathrm{Zn}^{2+}$ on tau phosphorylation appears to be bimodal, as at a concentration of $100 \mu \mathrm{M}$ the cation induces tau dephosphorylation. ${ }^{3}$ Furthermore, $\mathrm{Zn}^{2+}$ protects cells by blocking ion fluxes through $\mathrm{A} \beta$-induced cation permeable channels ${ }^{5}$ or competes with $\mathrm{Cu}^{2+}$ in its binding to $\mathrm{A} \beta$, thereby inhibiting amyloid plaque formation. ${ }^{6}$

$\mathrm{Zn}^{2+}$ is sequestered into the mitochondria ${ }^{7,8}$ and mitochondrial dysfunctions as well as energy deficiency are also prominent early events in AD. ${ }^{9}$ Furthermore, recent findings in a triple transgenic mouse model of $A D$ indicate a synergistic interaction between $\mathrm{A} \beta$ and $\mathrm{h}$-tau in impairing the oxidative phosphorylation system (OXPHOS) as well as the production of reactive oxygen species. ${ }^{9}$ In that respect, we have also previously shown the expression of pro-AD factors, such as mutant human presenilin-1 (PS1), amyloid precursor protein (APP) and human h-tau present in 3xTg-AD mice strongly alters intracellular $\mathrm{Zn}^{2+}$ homeostasis in cultured cortical neurons undergoing oxidative stress. ${ }^{10}$

Although it seems clear that alterations in the content of brain $\mathrm{Zn}^{2+}$ can have a pathogenic role in $\mathrm{AD},{ }^{2,11}$ there are still conflicting results about how the overall brain bioavailability of the cation can affect the disease progression. On one hand, several key studies have demonstrated that the use of $\mathrm{Zn}^{2+}$-binding compounds such as clioquinol or PBT2 restore $\mathrm{Zn}^{2+}$ homeostasis, greatly reduces $\mathrm{A} \beta$ pathology and protects against cognitive decline in transgenic $A D$ models. ${ }^{12,13}$ On the other hand, a recent study, in an $A D$ mouse model, ${ }^{14}$ has shown that dietary $\mathrm{Zn}^{2+}$ supplementation exacerbates cognitive deficits but surprisingly decreases amyloid deposits. Furthermore, $\mathrm{Zn}^{2+}$ depletion has been found to increase the volume of amyloid plaques ${ }^{15}$ and data obtained in transgenic mice genetically depleted of synaptic $\mathrm{Zn}^{2+}, \mathrm{ZnT3}-\mathrm{KO}$ mice, indicate that such depletion leads to an age-dependent decline of cognitive functions. ${ }^{16}$ Moreover, lactational zinc deficiency has been shown to promote apoptotic neuronal loss in the mouse hippocampus. ${ }^{17}$

\footnotetext{
${ }^{1}$ Molecular Neurology Unit-Center of Excellence on Aging (Ce.S.I.), University 'G. d'Annunzio', Chieti-Pescara, Italy; ${ }^{2}$ Department of Neuroscience and Imaging, University 'G. d'Annunzio', Chieti-Pescara, Italy; ${ }^{3}$ Department of Biological and Environmental Science, University of Sannio, Benevento, Italy; ${ }^{4}$ Department of Oncology and Neuroscience, University 'G. d'Annunzio', Chieti-Pescara, Italy; ${ }^{5}$ Department of Biomedical Science, University 'G. d'Annunzio', Chieti-Pescara, Italy and ${ }^{6}$ Department of Neurology, University of California-Irvine, Irvine, CA, USA

${ }^{*}$ Corresponding author: SL Sensi, Molecular Neurology Unit, Center of Excellence on Aging, University G. d'Annunzio, Chieti e Pescara, Via Colle dell' Ara, Chieti Scalo, 66013, Chieti, Italy. Tel: + 390871 541544; Fax: + 390871 541542; E-mail: ssensi@uci.edu

Keywords: Alzheimer's disease; $3 x T g-A D$ mouse; $A \beta$, tau; zinc; BDNF

Abbreviations: $\mathrm{Zn}^{2+}$, Zinc; $\mathrm{AD}$, Alzheimer's disease; $\mathrm{A} \beta, \beta$ amyloid; TrkB, tyrosine kinase receptor type B; BDNF, Brain-Derived Neurotrophic Factor; h-tau, hyperphosphorylated tau; OXPHOS, oxidative phosphorylation system; PS1, human presenilin-1; APP, Amyloid Precursor Protein; MMPs, matrix metalloproteinases; MWM, Morris Water Maze; NOR, Novel Object Recognition; BN-PAGE, Blue-Native Polyacrylamide gel electrophoresis; GSK-3 $\beta$, Glycogen synthase kinase-3- $\beta$; NMDA, N-Methyl-D-Aspartate; GABA, $\gamma$-Aminobutyric acid
}

Received 11.8.10; revised 20.9.10; accepted 27.9.10; Edited by V De Laurenzi 
Neurotrophic signaling pathways are also deregulated in AD. ${ }^{18}$ In particular, recent studies have shown that decrease levels of brain-derived neurotrophic factor (BDNF) correlate with the severity of AD-related cognitive impairment, ${ }^{19}$ suggesting that reduced BDNF availability may be an early cofactor involved in AD development. Interestingly, $\mathrm{Zn}^{2+}$ has been shown to be an important modulator of this pathway as the cation facilitates the maturation of BDNF from pro-BDNF through the activation of $\mathrm{Zn}^{2+}$-dependent matrix metalloproteinases (MMPs). ${ }^{20}$

In this study, we investigated the effect of dietary $\mathrm{Zn}^{2+}$ supplementation on the disease progression of the 3xTg-AD mouse, a transgenic animal model of AD that exhibits both $\mathrm{A} \beta$ - and tau-dependent pathology. $\mathrm{Zn}^{2+}$ supplementation was started early on in male animals at 1 month of age (m.o.a.) testing the hypothesis that such early exposure to the cation can set in motion a better intraneuronal $\mathrm{Zn}^{2+}$ homeostasis and counteract the aging-related $\mathrm{Zn}^{2+}$ deregulation that has been associated with $A D .^{2}$ End points of the study were analysis of cognitive decline, the appearance of intraneuronal $\mathrm{A} \beta$ and h-tau, $\mathrm{AD}$-related mitochondrial dysfunctions, as well as disruption of the BDNF neurotrophic pathway.

\section{Results}

Dietary zinc supplementation counteracts the development of hippocampus-dependent cognitive deficits in 3xTg-AD mice. To evaluate the role of $\mathrm{Zn}^{2+}$ supplementation in 3xTg-AD mice, 1-month-old male animals $(n=9)$ were treated with 30 p.p.m. of $\mathrm{ZnSO}_{4}$ for 11-13 months. PS1-KI mice $(n=11)$ that do not develop $\mathrm{A} \beta$ or tau pathology nor show any cognitive deficits were used as control animals. ${ }^{21}$ At the end of the treatment, mice were assessed for both hippocampus- and cortex-dependent cognitive tasks. Mice were first studied for their performance on the morris water maze (MWM) test, a task that is highly dependent on the hippocampus, to investigate spatial memory functioning. At first, we assessed the integrity of the mice learning process and found no differences in task acquisition (data not shown), indicating that, during a 3-day period of training, all groups learned equally well how to find the submerged platform using intra- and extra-maze visible cues. After the last training trial, spatial reference memory probe trials were conducted at 1.5 and $24 \mathrm{~h}$ to examine short- and long-term memory, respectively. As previously reported, ${ }^{21} 3 x T g-A D$ mice displayed no impairment in shortterm memory, while they manifested long-term memory deficits as indicated by the statistically significant increase in the time spent to find the platform (control versus 3xTg-AD; $(P=0.010))$. We also observed (even though it was found to be not statistically significant, $P=0.083)$, a trend toward decreased number of platform crosses (Figure 1a and b). Interestingly, $\mathrm{Zn}^{2+}$ treatment prevented the long-term memory decline in 3xTg-AD mice as indicated by key MWM parameters such as the latency to find the platform and the number of platform crosses that were basically identical to the ones shown by disease-free PS1-KI mice ((Figure 1a and b); 3xTg-AD versus $3 \times T_{g}-\mathrm{AD}+\mathrm{Zn}^{2+}$,

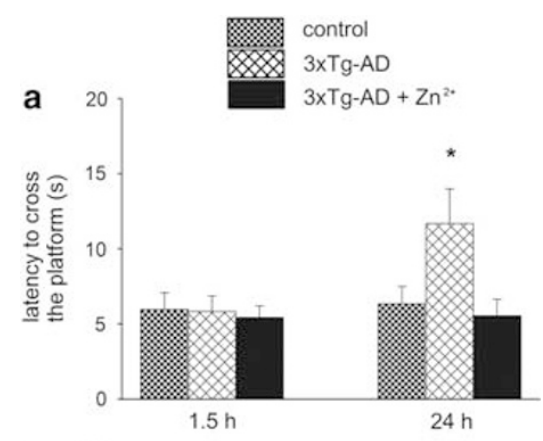

b
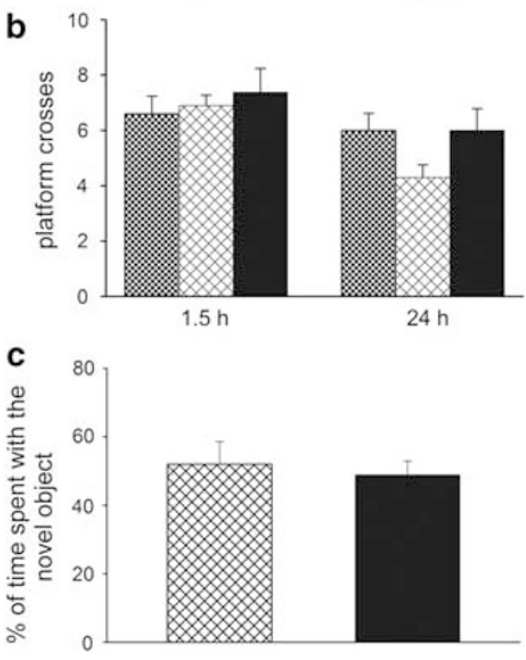

Figure $1 \mathrm{Zn}^{2+}$ supplementation prevents the development of hippocampusdependent memory deficits in 3xTg-AD mice. Male 3xTg-AD mice were treated with either water containing 30 p.p.m. of $\mathrm{ZnSO}_{4}(n=9)$ or tap water $(n=11)$ for $11-13$ months and tested for the spatial memory version of the MWM. (a and $\mathbf{b}$ ) Mice were given a memory probe trial wherein, the platform was removed 1.5 and $24 \mathrm{~h}$ after the last training trial. Compared with untreated $3 \times T$ Tg-AD mice, $\mathrm{Zn}^{2+}$-fed $3 \times \mathrm{Tg}$-AD mice do not show any significant difference in their MWM performance at $1.5 \mathrm{~h}$. (a) When tested at $24 \mathrm{~h}$, mice exhibit a marked decrease in the time (latency) they employed to reach the point where the platform used to be. (b) $\mathrm{Zn}^{2+}$-treated $3 \times \mathrm{Tg}$-AD mice make also more platform crosses, even though such parameter does not reach statistical significance. (c) $\mathrm{Zn}^{2+}$ treatment does not affect the performance of the cortex-dependent novel object recognition test as treated and untreated 3xTg-AD mice show no differences in their ability to remember an object to which they were exposed $24 \mathrm{~h}$ before. Error bars indicate mean values \pm S.E.M.; ${ }^{\star} P<0.05$

$P=0.011)$. We next evaluated the effect of $\mathrm{Zn}^{2+}$ supplementation on the novel object recognition (NOR) test, a cortex-dependent contextual task which relies on the animal preference to explore a novel object over a familiar object. $^{22-24}$ Analysis of the NOR performance showed no differences between treated and untreated 3xTg-AD mice (Figure 1c; $(P=0.669))$ indicating that the beneficial effects of $\mathrm{Zn}^{2+}$ were region-specific and restricted to the hippocampus.

Zinc supplementation reduces intraneuronal $A \beta$ and tau pathology in the hippocampus of 3xTg-AD mice. After the cognitive evaluation, mice were killed and neuropathology assessed to elucidate the underlying mechanisms by which $\mathrm{Zn}^{2+}$ feeding had improved cognition. 3xTg-AD mice develop a progressive intraneuronal $\mathrm{A} \beta$ accumulation in AD-relevant 
regions such as the cerebral cortex and the hippocampus starting at 4 m.o.a. ${ }^{25}$ To determine whether $\mathrm{Zn}^{2+}$ supplementation can decrease the brain $A \beta$ load, immunohistochemistry was performed with the anti-A $\beta$ DE2B4 primary antibody and results of this assay show a significant decrease in the hippocampus (Figure 2a-e; $(P=0.013)$ ), but not in the cortex of treated 3xTg-AD mice (see supplementary Figure $1 \mathrm{a}$ and $\mathrm{b}$ ), a finding that matches their behavioral performance.

At about 12 m.o.a., 3xTg-AD mice also show extensive neurofibrillary tangles, first in the hippocampus (in particular within pyramidal neurons of the CA1 subfield) and then in the cortex. ${ }^{25}$ To investigate the effect of our $\mathrm{Zn}^{2+}$ treatment on the development of tau pathology, we employed an anti-tau AT180 primary antibody that specifically detects tau phosphorylation at the thr231/ser235 site. As expected, we found diffuse h-tau immunoreactivity in the CA1 subfield and cortex of untreated 3xTg-AD mice (Figure $2 f$ and $g$ ). Interestingly, $\mathrm{Zn}^{2+}$ treatment produced a marked decrease of $\mathrm{h}$-tau immunoreactivity in the $3 \times \mathrm{Tg}-\mathrm{AD}$ CA1 subfield (Figure $2 \mathrm{~h}-\mathrm{j}$;
$(P=0.010))$ but not in the cortex (see supplementary Figure $1 \mathrm{c}$ and d), further indicating that $\mathrm{Zn}^{2+}$ supplementation has a potent region-specific effect on tau pathology.

Zinc supplementation restores mitochondrial function in 3xTg-AD mice. Mitochondrial dysfunctions have a pivotal role in $A D^{9}$ and a previous study in 3xTg-AD mice has shown that such deficits are developed at early stages and precede the manifestation of amyloid- and tau-dependent pathology. ${ }^{26}$ To investigate the effect of our $\mathrm{Zn}^{2+}$ treatment on AD-related mitochondrial deficits, we investigated the activity of mitochondrial complexes by employing a combination of blue-native polyacrylamide gel electrophoresis (BN-PAGE) and subsequent histochemical in-gel staining. As first, to evaluate a potential age-dependent decline in mitochondrial respiration, we studied the activity of mitochondrial complexes I, II and IV obtained from homogenates of the cortex, hippocampus and cerebellum of 3xTg-AD mice at 2, 6 and 12-14 m.o.a. These activities were compared with age-matched PS1-KI mice. In the case
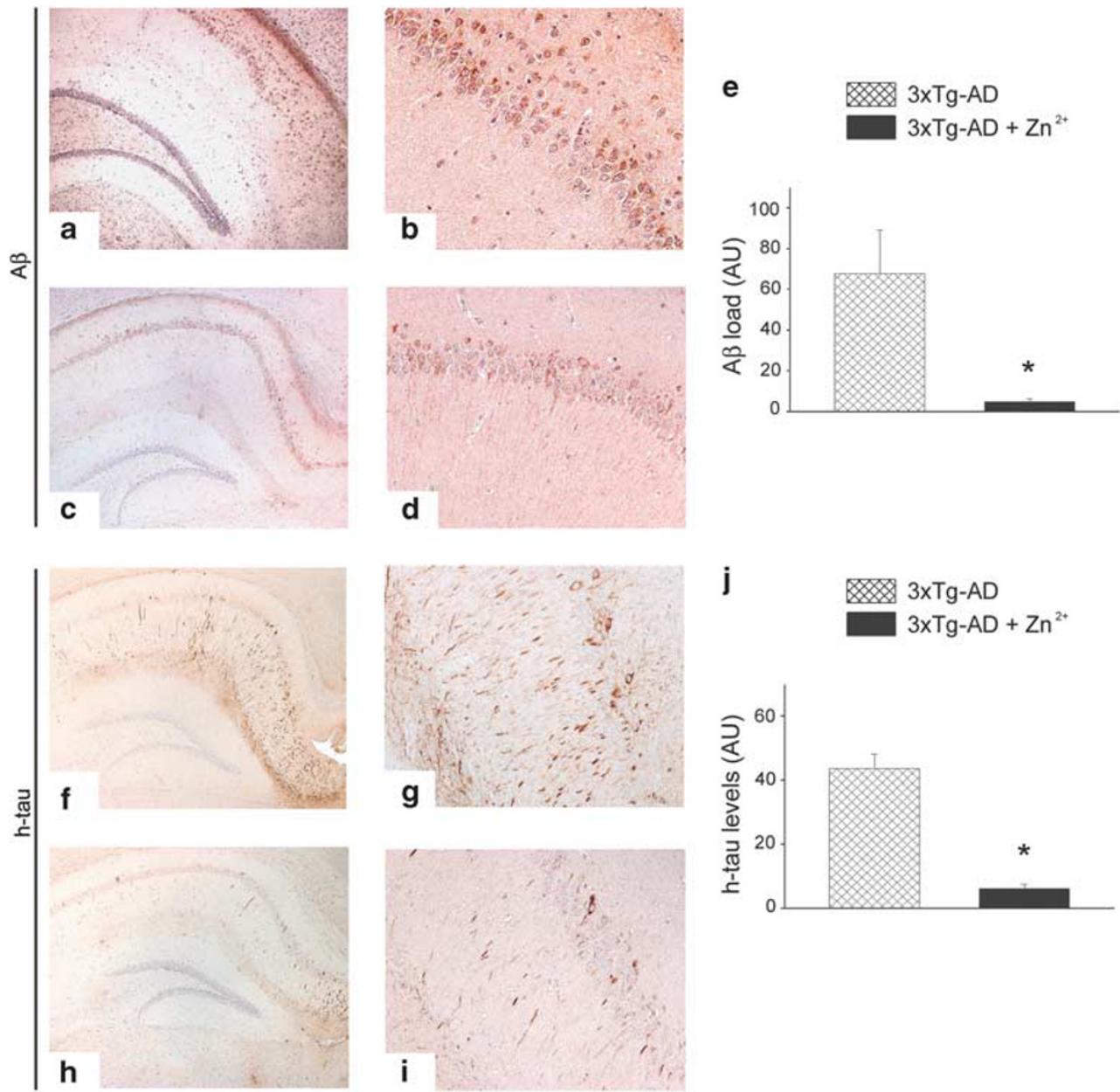

Figure $2 \mathrm{Zn}^{2+}$ supplementation reduces both $\mathrm{A} \beta$ and tau pathology in the hippocampus of $3 \mathrm{xTg}-\mathrm{AD}$ mice. Immunohistochemistry with specific antibodies was employed to detect deposits of intraneuronal $\mathrm{A} \beta(\mathbf{a}-\mathbf{d})$ and $\mathrm{h}$-tau $(\mathbf{f}-\mathrm{j})$ in brain slices from treated and untreated $3 \times \mathrm{Tg}-\mathrm{AD}$ mice (left column: $20 \times$ magnification; right column: $40 \times$ magnification). Compared with untreated mice (a and $\mathbf{b}$ ), DAB staining shows a significant decrease of intraneuronal $A \beta$ deposits in the hippocampus of treated $3 \times T$ Tg-AD mice (c and d). (b and d) $40 \times$ magnification of the hippocampal CA1 area as shown in the rectangle. (e) Quantification of A $\beta$ load as shown in a and $\mathbf{c}$. Compared with untreated mice (f, $\mathbf{g})$, DAB staining shows a strong decrease of intraneuronal $\mathrm{h}$-tau immunoreactivity in the hippocampus of $\mathrm{Zn}{ }^{2+}-f e d$ 3xTg-AD mice $(\mathbf{h}$ and $\mathbf{i}) .(\mathbf{g}$ and $\mathbf{i})$ $40 \times$ magnification of the CA1 area as shown in the rectangle. (j) Quantification of $\mathrm{h}$-tau levels from $\mathrm{f}$ and $\mathrm{h}$. Error bars indicate mean values $\pm S$.E.M.; ${ }^{*} P<0.05$ 


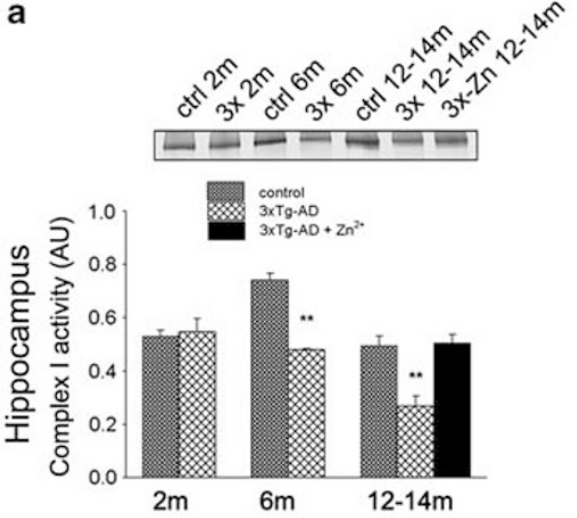

d
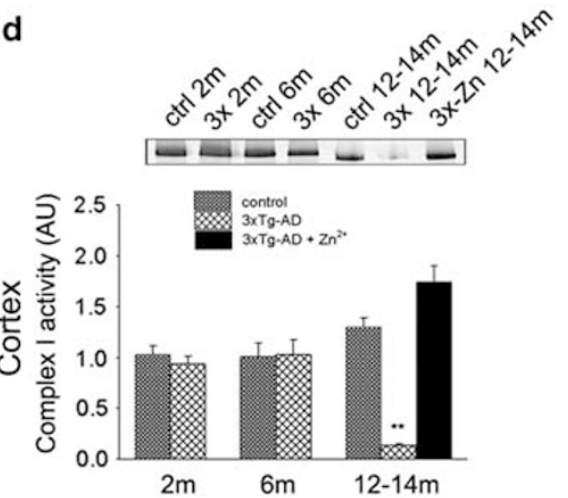

g
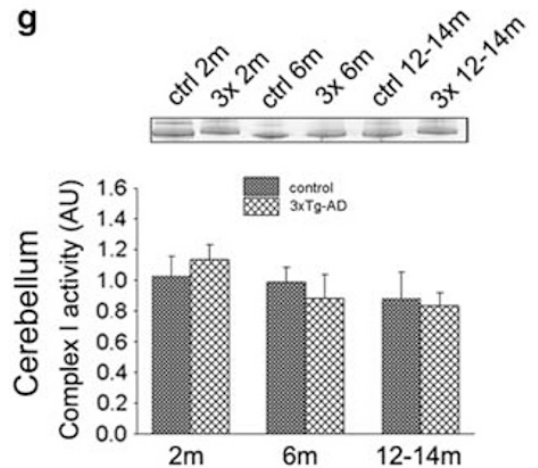

b

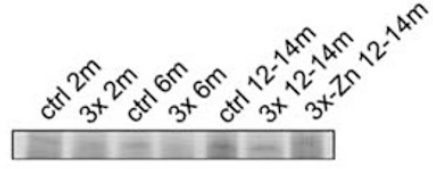

C
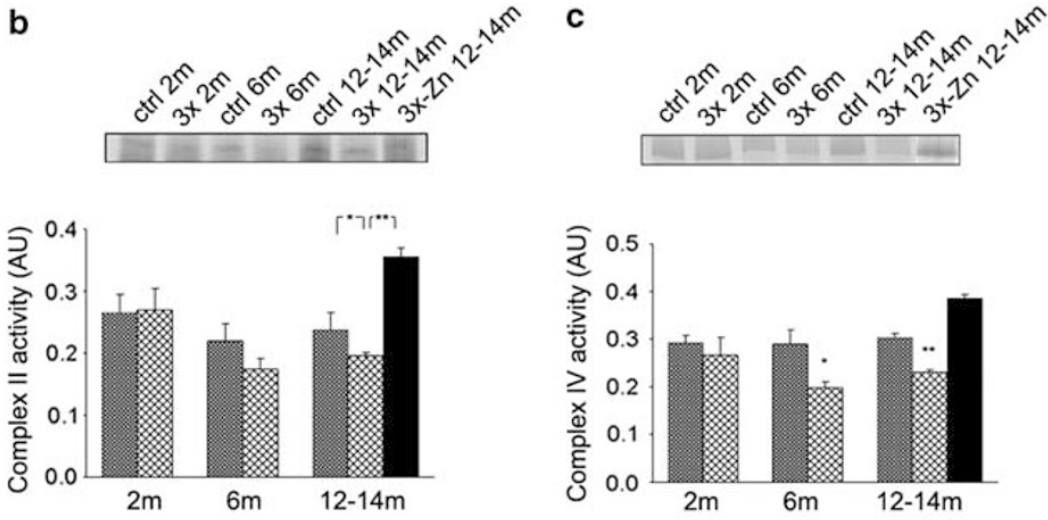

e

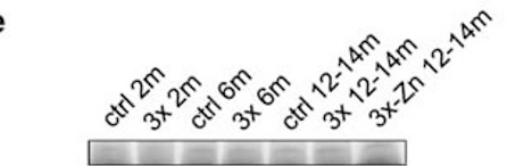

f
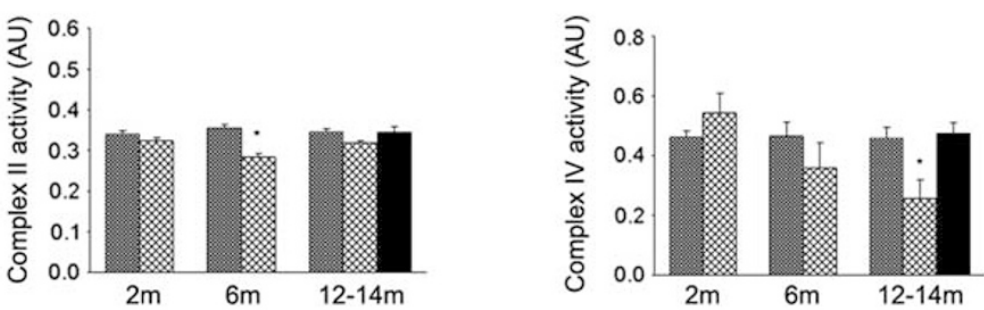

h

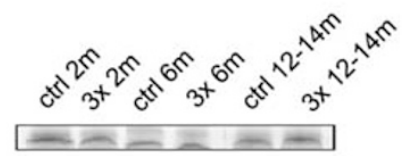

i
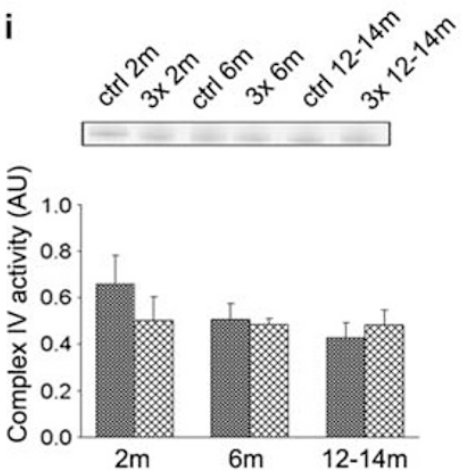

Figure $3 \mathrm{Zn}^{2+}$ supplementation rescues AD-related mitochondria deficits in 3xTg-AD mice. BN-PAGE was employed to assess the activity of mitochondrial complexes I $(\mathbf{a}, \mathbf{d}$ and $\mathbf{g}), \boldsymbol{I I}(\mathbf{b}, \mathbf{e}$ and $\mathbf{h})$ and IV (c, $\mathbf{f}$ and $\mathbf{i})$ in isolated mitochondria obtained from the hippocampus (a-c), cerebral cortex (d-f), or the cerebellum ( $\mathbf{g}-\mathbf{i})$ of treated and untreated 3xTg-AD, as well as control mice at 2, 6 and 12-14 m.o.a. When compared with age-matched untreated mice, functioning of mitochondrial complexes I and IV appears to be decreased in the hippocampus of untreated 6-month-old 3xTg-AD mice (a and $\mathbf{c}$ ). The same deficits are found in hippocampal mitochondria of 3xTg-AD mice at 12-14 m.o.a.; however, $\mathrm{Zn}^{2+}$ supplementation completely counteracts the development of these deficits (a-c). When comparing complex activities of cortical mitochondria obtained from 6-month-old 3xTg-AD versus age-matched control mice, complexes I and IV are found not compromised (d-f) while a statistically significant deficit is observed in the case of complex II (e). Still in the cortex, complexes I and IV functioning is greatly compromised in untreated $3 x \mathrm{Tg}-\mathrm{AD}$ mice at 12-14 m.0.a. and $\mathrm{Zn} \mathrm{n}^{2+}$ treatment is able to completely rescue the deficits (d-f). No changes are found in the cerebellum of $3 \times \mathrm{Tg}-\mathrm{AD}$ mice compared with age-matched controls $(\mathbf{g}-\mathbf{i})$. ${ }^{*} P<0.05 ;{ }^{* *} P<0.01$. Error bars indicate mean values \pm S.E.M.

of experiments with mice at 2 and 6 m.o.a., we employed a pool of three-five animals for each age group and each genotype. We then compared the activities of these mitochondrial complexes in treated and untreated 3xTg-AD mice at $12-14$ m.o.a.

Hippocampus. When comparing 3xTg-AD with control mice, we confirmed the impairment in mitochondrial function described by Yao et al. ${ }^{26}$ and found an age-dependent decay in the activity of mitochondrial complexes I and IV starting at 6 m.o.a. (Figure $3 a-c$; (control versus $3 x T g-A D$ : Complex I, $P=0.010$; Complex II, $P=0.048$; Complex IV, $P \ll 0.01)$ ). Changes in enzyme activities were not dependent on changes in protein expression (data not shown). $\mathrm{Zn}^{2+}$-supplemented 3xTg-AD mice at 12-14 m.o.a. showed a complete recovery of all these defective respiratory 
complexes (Figure 3a-c; (3xTg-AD versus $3 x T g-A D+\mathrm{Zn}^{2+}$ : Complex I, $P=0.008$; Complex II, $P=0.001$; Complex IV, $P \ll 0.01)$ ).

Cortex. In contrast to what was found in the hippocampus, compared with control mice 3xTg-AD animals showed no deficits at 2 and 6 m.o.a., except for the complex II at 6 m.o.a. $(P=0.005)$. Moreover, activities of complexes I and IV were also dramatically reduced at 14 m.o.a. (control versus 3xTg-AD: Complex I, $P \ll 0.01$; Complex IV, $P=0.047$ ). Notably, $\mathrm{Zn}^{2+}$-supplemented 3xTg-AD mice at 12-14 m.o.a. showed a complete recovery in the activity of both complexes I and IV (Figure 3d-f; (3xTg-AD versus $3 x T g-A D+\mathrm{Zn}^{2+}$ : Complex I, $P \ll 0.01$; Complex IV $P=0.045)$ ).

Cerebellum. To test whether the mitochondrial deficits observed in the cortex and hippocampus were due to $A D$-related mechanisms triggered by the expression of mutant APP, PS1 and h-tau, we also analyzed the activity of complexes I, II and IV in the cerebellum, a region that shows no $\mathrm{A} \beta$ - or tau-dependent pathology and is not affected by $A D$. As expected, mitochondrial respiratory activities of all the complexes showed a not statistically significant agedependent decline in $3 x \operatorname{tg}-\mathrm{AD}$ mice, compared with control mice (Figure $3 g-i$ ).

Dietary zinc supplementation increases BDNF levels in 3xTg-AD brains through MMPs activation. BDNF signaling pathway is downregulated in $A D,{ }^{18}$ and $\mathrm{Zn}^{2+}$ has been described to potently stimulate the maturation of BDNF from inactive pro-BDNF via MMP activation. ${ }^{20}$ We hence employed gelatine zymography assay in order to investigate the effect of $\mathrm{Zn}^{2+}$ supplementation on the activation of gelatine-degrading MMP-2 and MMP-9 in treated and untreated 3xTg-AD mice brains. As shown in Figure 4a, $\mathrm{Zn}^{2+}$ treatment potently increased both MMP-2 and MMP-9 activity compared with untreated 3xTg-AD mice $(P=0.004$ and $P=0.001$, respectively). Finally, we evaluated the effect of $\mathrm{Zn}^{2+}$ supplementation on cerebral BDNF levels by performing immunoblotting assay on treated and untreated
3xTg-AD brains. As shown in Figure $4 b, \mathrm{Zn}^{2+}$ supplementation induced a fourfold increase in BDNF brain levels $(P=0.022)$.

\section{Discussion}

Although intraneuronal $\left(\mathrm{Zn}^{2+}\right)$ rises are toxic, $\mathrm{Zn}^{2+}$ is required for many physiological cell functions. ${ }^{2} \mathrm{Zn}^{2+}$ is an essential nutritional factor and its deficiency may have important pathological consequences. ${ }^{27}$ In that respect, recent reports have estimated that more than $25 \%$ of the world population is at risk of $\mathrm{Zn}^{2+}$ deficiency. ${ }^{28,29}$ In the context of $\mathrm{AD}$, however, studies on $\mathrm{Zn}^{2+}$ deficiency or supplementation in $A D$ models have provided conflicting results. ${ }^{14,15,30}$ Here, we show that dietary $\mathrm{Zn}^{2+}$ supplementation prevents hippocampal-dependent cognitive deficits, reduces both intraneuronal $A \beta$ and tau pathology, prevents mitochondrial deficits and restores BDNF levels in a mouse model of AD.

Furthermore, as the gender difference has an important role in the pathology progression as well as in the $\mathrm{Zn}^{2+}$ homeostasis of $A D$ mice, ${ }^{31,32}$ we decided to test our treatment in male 3xTg-AD and PS1-KI mice in order to avoid any confounding effect driven by female hormones.

In the last years, several lines of evidence indicate that the accumulation of $A \beta$ in the intraneuronal compartment, more than the extracellular space, is crucial in promoting the amyloid pathogenic effects. ${ }^{33}$ Our mouse model exhibits an intraneuronal $\mathrm{A} \beta$ accumulation in the hippocampus and cortex that correlates with synaptic and LTP dysfunctions as well as with the development of memory deficits. ${ }^{21,34}$ Here we show that $\mathrm{Zn}^{2+}$ supplementation in $3 \mathrm{xTg}-\mathrm{AD}$ mice strongly reduces the intraneuronal $A \beta$ deposition in the hippocampus but not in the cerebral cortex.

Furthermore, recent observations describe a dose-dependent bimodal action exerted by the cation on tau phosphorylation and conformation. ${ }^{3}$ High $\mathrm{Zn}^{2+}$ concentrations $(250 \mu \mathrm{M})$ induce increased tau phosphorylation via glycogen synthase kinase-3- $\beta$ activation, while low- $\mathrm{Zn}^{2+}$ concentrations $(\sim 100 \mu \mathrm{M})$ promotes tau dephosphorylation of the PHF-1

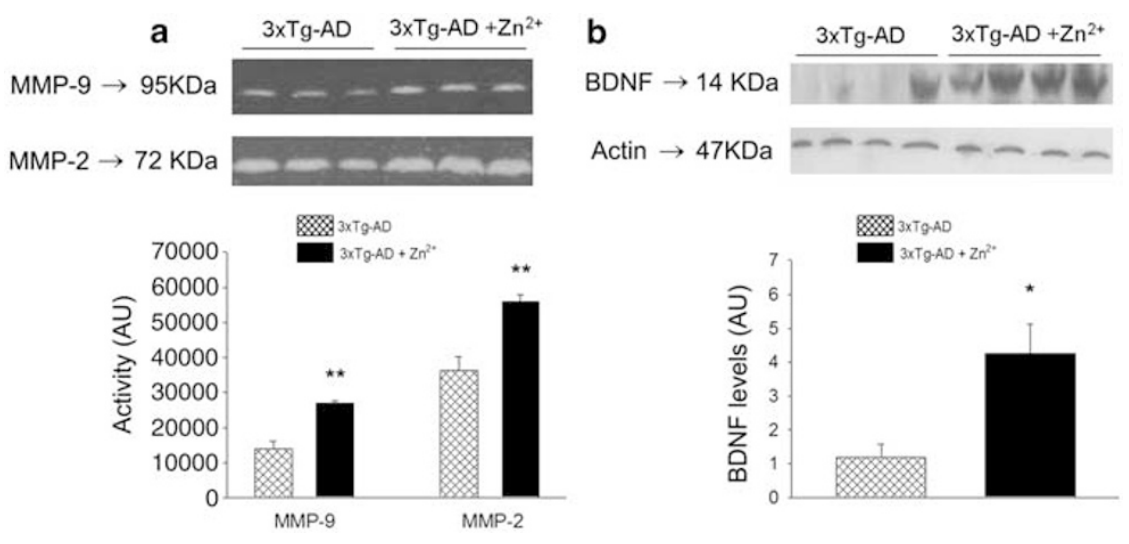

Figure $4 \mathrm{Zn}^{2+}$ supplementation promotes MMPs activation and increases BDNF levels in 3xTg-AD mice. (a) Gelatine zymography indicates that $Z n^{2+}$ treatment induces a significant increase of MMP-2 and MMP-9 activation in 3xTg-AD mice brains. (b) BNDF immunoblotting reveals that $Z \mathrm{n}^{2+}$-treated $3 \times \mathrm{Tg}$-AD mice show a fourfold increase in BDNF levels when compared with untreated animals. ${ }^{*} P<0.05 ;{ }^{*} P<0.01$. Error bars indicate mean values \pm S.E.M. 
epitope. Our data strongly support this line of evidence as we find that 30 p.p.m. (equivalent to $460 \mu \mathrm{M}$ ) of $\mathrm{Zn}^{2+}$ induces a significant reduction of tau phosphorylation at the thr231/ ser235 site of CA1 hippocampal pyramidal neurons. The fact that the $\mathrm{Zn}^{2+}$ concentration we used to feed our animals is higher than $250 \mu \mathrm{M}$, but still able to reduce the hippocampal tau pathology might be explained by the filtering activity of the blood brain barrier that makes the final $\mathrm{Zn}^{2+}$ concentration in the brain parenchyma likely comparable to the 'low' $\mathrm{Zn}^{2+}$ levels employed in culture studies.

Complementary to such region-specific decrease of both $\mathrm{A} \beta$ and tau-dependent pathology, our treatment also completely prevents hippocampus-dependent spatial memory deficits while not affecting a cortex-dependent cognitive test. Although the mechanisms producing these regional differences are unknown, one possible explanation may be associated with a distinct difference between the hippocampus and the cortex in $\mathrm{A} \beta$ and h-tau load.

Our results are not in accordance with what was reported by Linkous et al. ${ }^{14}$ as they found decreased amyloid plaque deposition, but increased cognitive deficits in $\mathrm{Zn}^{2+}$-fed Tg2576 and TgCRND8 mice. One possible explanation for such discrepancy can be related to the difference in the animal models employed in the two studies. The 3xTg-AD mice manifest signs of $A \beta$ and tau pathology and cognitive deficits that are highly dependent on the intraneuronal deposition of $\mathrm{A} \beta^{21}$ whereas the mice used by Linkous et al. show memory deficits mainly dependent on extracellular amyloid overload. The different pathogenic weight of extracellular versus intracellular amyloid deposition as well as the injurious interaction between amyloid and tau present in the 3xTg-AD model but not in Tg2576 and TgCRND8 mice, may likely explain the divergent effects we report.

Energy deficiency and mitochondrial dysfunctions are linked to $A D$, and recent findings describe the synergistic interaction between $\mathrm{A} \beta$ and tau in promoting OXPHOS impairment in $A D$ mice. ${ }^{9}$ In line with these findings, we find a significant decay in the activity of mitochondrial complexes I, II and IV in the hippocampus and of complexes I and IV in the cerebral cortex of aging 3xTg-AD mice, although no changes are found in their cerebellum. Notably, all the mitochondrial deficits are prevented by $\mathrm{Zn}^{2}+$ supplementation.

Tau has been shown to specifically impair mitochondrial complex I activity. ${ }^{9}$ Here we show that complex I functioning is reduced in the hippocampus of aging $3 x T g-A D$ mice and $\mathrm{Zn}^{2+}$ rescues the complex activity in parallel with a dramatic decrease in h-tau hippocampal immunoreactivity. In the cerebral cortex, complex I activity shows a dramatic decrease and such deficit is prevented by $\mathrm{Zn}^{2+}$ even though cortical tau immunoreactivity is not changed, thereby suggesting that additional region-specific factors are playing a role in promoting mitochondrial dysfunction in our model. Complex II activity shows a significant reduction in the hippocampus while it appears to be unchanged in cortical areas, with the exception of animals at 6 m.o.a. Finally, we observe a decrease in the activity of mitochondrial complex IV in the hippocampus and cortex, a phenomenon that has been previously shown to be deregulated by both $A \beta$ and tau in $A D$ models. ${ }^{9}$ Our data confirm these findings as we show that the strong $\mathrm{A} \beta$ and tau immunoreactivity we observed in these areas goes along with a great decay of complex IV activity. Notably, $\mathrm{Zn}^{2+}$-treated 3xTg-AD mice show a complete recovery of mitochondrial complex IV activity in both the hippocampus and cerebral cortex. Taken together, our cortical data seem to indicate that mitochondria dysfunctions have a partial role in promoting AD-related cognitive deficits, as simply restoring the activity of OXPHOS complexes is per se not sufficient to induce a significant amelioration of cognitive functions.

One possible mechanism by which $\mathrm{Zn}^{2+}$ is beneficial in our $A D$ model is offered by its modulation of the BDNF-tyrosine kinase receptor type $B$ (TrkB) receptor pathway. BDNF is produced as a biologically inactive form (pro-BDNF) but once converted to mature BDNF, has a pivotal role not only in neuronal survival and differentiation, but also in maintaining synaptic structure, function and plasticity. ${ }^{35}$ Altered BDNF signaling has been shown to be involved in several neurodegenerative disorders including $A D .{ }^{36} \mathrm{Zn}^{2+}$ induces BDNF maturation from pro-BDNF through MMPs activation and also transactivates synaptic TrkB by promoting Src family kinase activity. ${ }^{37}$ In our study, we find a strong decrease of BDNF levels in aging 3xTg-AD mice compared with agematched control mice (data not shown). Interestingly, $\mathrm{Zn}^{2+}$ supplementation induces a strong potentiation of MMP-2 and MMP-9 activity that leads to a fourfold increase of BDNF levels. Our results parallel findings of a study that employed ZnT3-KO mice that lack synaptic $\mathrm{Zn}^{2+}$, show signs of cognitive impairment and have decreased BDNF and TrkB levels. ${ }^{16}$ Indeed, the fact that $\mathrm{Zn}^{2+}$ supplementation is beneficial in rescuing hippocampal dysfunctions seems to further substantiate the pivotal role played by synaptic $\mathrm{Zn}^{2+}$ in shaping the physiological neurotransmission of this crucial brain region. The cation can critically modulate long-term potentiation and depression by interfering with the functioning of $N$-methyl-D-aspartate and $\gamma$-aminobutyric acid receptors and recent findings also indicate that $\mathrm{Zn}^{2+}$ can act on the $\mathrm{ZnR}$, a post-synaptic metabotropic $\mathrm{Zn}^{2+}$-sensing receptor, which activates MAPK and CAMK2 and ultimately affects synaptic plasticity. ${ }^{2,38}$ Finally, as MMPs have a role in degrading $\mathrm{A} \beta{ }^{39,40}$ we can speculate that the $\mathrm{Zn}^{2+}$-triggered increase of MMP activity might also exert a critical neuroprotective effect by potentiating the clearance of intraneuronal amyloid deposits.

In summary, our data lend support to the hypothesis that a fine-tuning of brain $\mathrm{Zn}^{2+}$ homeostasis may be beneficial in the treatment of $A D$.

\footnotetext{
Materials and Methods

Animals and treatment paradigm. Procedures involving animals and their care were conducted in conformity with the institutional guidelines that are in compliance with national (D.L. n. 116, G.U., suppl. 40, 18 February 1992) and international laws and policies. All efforts were made to minimize the number of animals used and their suffering. Transgenic mice were generated by Oddo et al. ${ }^{34}$ and generously provided by Frank Laferla. Tap water containing zinc sulfate $\left(\mathrm{ZnSO}_{4}\right)$ was fed to 1-month-old male $3 \times \mathrm{Tg}-\mathrm{AD}$ mice $(n=9)$ for a period of $11-13$ months. Age-matched untreated $3 \times \mathrm{Tg}-\mathrm{AD}(n=9)$ or PS1-KI $(n=11)$ mice were considered controls and fed with tap water. For the $\mathrm{Zn}^{2+}$ treatment, $132 \mathrm{mg} / \mathrm{l}$ of $\mathrm{ZnSO}_{4}$ were added to tap water to reach a final concentration of $30 \mathrm{mg} / \mathrm{l}$ of $\mathrm{Zn}^{2+}$ (30 p.p.m.). The stability of the $\mathrm{Zn}^{2+}$-enriched solution was verified by inductively coupled plasma mass spectrometry.
} 


\section{Cognitive tasks}

MWM. The MWM apparatus consisted of a circular plastic tank ( $1.3 \mathrm{~m}$ diameter) filled with water. The maze was located in a room containing several intra and extra-maze visual cues. A visible platform was employed at the beginning of the MWM test to make sure animals had no vision deficits. Mice were then trained to swim in the tank and climb on a $(12 \times 13 \mathrm{~cm})$ rectangular platform submerged $2 \mathrm{~cm}$ beneath the surface of the water. No differences in the swimming speed were observed between groups. To reduce the stress related to the task, mice were placed on the platform $10 \mathrm{~s}$ before the beginning of the first training session. If a mouse failed to find the platform within $90 \mathrm{~s}$, it was manually guided to the platform and allowed to remain there for $10 \mathrm{~s}$. Mice were placed, between trials, back into a holding cage under a warming fan for $20 \mathrm{~min}$. Mice were given four trials per day for 3 consecutive days. Retention of the spatial memory task was assessed at 1.5 and $24 \mathrm{~h}$ after the end of the last training trial. Both probe trials consisted of a $60 \mathrm{~s}$ free swim in the pool without the platform. The parameters measured during the probe trial included: (1) the time (latency) spent to reach and cross the platform location and (2) the number of crosses over the platform location. Mice were tested at 12-14 m.o.a. and killed immediately after completion of the test.

NOR. Two plexiglass $(30 \times 15 \mathrm{~cm})$ cages were used for the whole experiment. In the habituation phase, mice, one per cage, were placed for 15 min per day for 2 consecutive days in empty cages. On day 3 , the mouse was placed in a cage containing two identical objects spaced $\sim 15 \mathrm{~cm}$ apart and allowed to explore the objects for $8 \mathrm{~min}$ before returning to the home cage. To rule out innate preferences, all the objects (made of plastic and of 5-6 cm in height) were tested on mice that were not part of the study. Objects were washed thoroughly with $70 \%$ ethanol before each trial. On day 4, the mouse was returned to the experimental cage containing the object to which it was previously exposed (familiar object), as well as a new object (novel object) positioned as the day before. Mice were then allowed to explore familiar and novel objects during a 5 min-probe test that was videotaped for subsequent analysis. Scoring of the NOR performance was based on the time spent to explore both familiar and novel objects. The behavior of the mouse was considered explorative (i.e. exploration) when the animal head was within $1 \mathrm{~cm}$ from the object with the neck extended and vibrissae moving. Simple proximity, chewing, or standing on the object did not count as exploration. Novel object exploration was calculated, as percentage, applying the following formula: $[A /(A+B)] \times 100$, where $A$ is the time spent to explore the novel object and $B$ is the time spent on the familiar one. As with the MWM test, mice were tested at 12-14 m.o.a. and killed immediately after completion of the test.

Brain tissue preparation. Mice were killed by carbon dioxide inhalation, and brains rapidly taken after removal of cerebellum and sagittally halved. One hemisphere and the cerebellum were frozen in liquid nitrogen and stored at $-80^{\circ} \mathrm{C}$ until use for further western blotting analysis, the other hemisphere was included for $24-36 \mathrm{~h}$ at room temperature (RT) in Carnoy fixative solution (65\% ethanol and $35 \%$ chloroform) and eventually processed for immunohistochemistry. In the case of BN-PAGE analysis, brains were quickly dissected on ice and the cerebellum, cortex and hippocampus immediately frozen on dry ice and stored at $-80^{\circ} \mathrm{C}$.

Immunoblotting. Tissue samples were homogenized in an ice-cold lysis buffer ( $50 \mathrm{mM}$ Tris- $\mathrm{HCl}, \mathrm{pH} 8,150 \mathrm{mM} \mathrm{NaCl}, 0.1 \% \mathrm{SDS}, 0.5 \mathrm{mM}$ sodium orthovanadate, $1 \mathrm{mM}$ sodium fluoride and $1 \%$ Triton $\mathrm{X}-100$ ) supplemented with a cocktail of protease and phosphatase inhibitors (Sigma-Aldrich, St Louis, MO, USA). The homogenates were briefly sonicated and then centrifuged at $4^{\circ} \mathrm{C}$ for $30 \mathrm{~min}$ at 14000 r.p.m. The supernatant was used for immunoblot analysis. Protein concentration was determined using the Bradford method (Bio-Rad, Hercules, CA, USA). In all, $60-80 \mu \mathrm{g}$ of proteins was resolved by SDS-PAGE (8-12\% Bis-Tris from Sigma-Aldrich) under reducing conditions and transferred to a nitrocellulose membrane. The membrane was incubated in Tris-buffered saline and Tween 20 (TBST) containing $5 \%$ of nonfat dry milk for $1 \mathrm{~h}$ at $20^{\circ} \mathrm{C}$. After an overnight incubation at $4^{\circ} \mathrm{C}$ in TBST with the primary antibody, blots were washed for $30 \mathrm{~min}$, incubated for an additional hour at $20^{\circ} \mathrm{C}$ with the secondary antibody and finally developed using the ECL PLUS (GE Healthcare, Little Chalfont, UK) chemiluminescence system. Bands were quantified by densitometry using NIH- Image $\mathrm{J} 1.61$ (public domain software).

Antibodies. The following antibodies were used: anti-A $\beta$, clone DE2B4, diluted 1:400 (Abcam, Cambridge, MA, USA); anti-phosphoTau AT180 1:400 (Pierce, Rockford, IL, USA); anti- $\beta$-actin 1: 10000 (Sigma-Aldrich) and anti-BDNF, $1: 1000$ (Cell Signaling, Beverly, MA, USA). Secondary ECL antibodies both anti-mouse and anti-rabbit (GE Healthcare) peroxidase linked were also used.
Immunohistochemistry. Carnoy-fixed and paraffin embedded brains were sagittaly sectioned ( $n=5$ per group). Sections were not pretreated with formic acid. Antigen retrieval was performed by microwave treatment at $750 \mathrm{~W}$ for $10 \mathrm{~min}$ in a $10 \mathrm{mmol} / \mathrm{l}$ sodium citrate buffer ( $\mathrm{pH}$ 6.0). After blocking the endogenous mouse lgG antibodies (Biocare Medical, Concord, CA, USA), sections were incubated overnight with the primary antibody. Slices were then incubated with the secondary anti-mouse (HRP-polymer EnVision kit, Dako, Glostrup, Denmark), counterstained with Mayer's hematoxylin and the reaction visualized using diamminobenzidine as chromogen. Images of $h$-tau and $A \beta$ stained sections were acquired using a $100 \times$ (for $A \beta$ ) or $200 \times$ (for h-tau) magnifications with a Leica DMR microscope (Leica Microsystems GmbH, Wetzlar, Germany) equipped with a DMR 500 digital camera (Leica). The number of stained pyramidal neurons and neurofibrillary tangles was measured using Photoshop 8.0 (Adobe Systems Incorporated, San Jose, CA, USA) by using the Photoshop Lasso Tool and pixel numbers obtained from the resulting histogram. After this first step, we used the Magic Wand tool to select a representative positive cell signal. All the immunostained cells were automatically selected and the total pixel number recorded. Pixel counts were normalized to a hippocampal area of $1 \mathrm{~mm}^{2}$

BN-PAGE on mitochondrial samples. After solubilization of mitochondrial membranes by dodecyl maltoside, $20 \mu \mathrm{g}$ of each sample was loaded on a $6-13 \%$ gradient acrylamide gel and subjected to electrophoresis on ice. For each set of gels, one gel was stained with Coomassie blue and the second one used to determine enzymatic activities. Complex I (NADH-dehydrogenase) activity was determined by incubating the gel with $0.1 \mathrm{M}$ Tris- $\mathrm{HCl}, 768 \mathrm{mM}$ glycine, $0.1 \mathrm{mM}$ $\beta$-NADH and $0.04 \%$ nitro blue tetrazolium at $\mathrm{pH} 7.4$ and RT. As an index of complex II activity, we evaluated succinate dehydrogenase activity incubating the gel in $0.1 \mathrm{M}$ Tris- $\mathrm{HCl}, 100 \mathrm{mM}$ glycine, $10 \mathrm{mM}$ succinic acid and $1 \mathrm{mg} / \mathrm{ml} \mathrm{NTB}$ at pH 7.4 and RT. Complex IV (COX) activity was estimated by incubating BN-PAGE gels with $5 \mathrm{mg}$ $3,3^{\prime}$-diaminobenzidine tetrahydrochloride (DAB) dissolved in $9 \mathrm{ml}$ phosphate buffer $(0.05 \mathrm{M}, \mathrm{pH} 7.4), 1 \mathrm{ml}$ catalase $(20 \mu \mathrm{g} / \mathrm{ml}), 10 \mathrm{mg}$ cytochrome $\mathrm{C}$ and $750 \mathrm{mg}$ sucrose.

Violet-colored complex I and II bands and red-stained complex IV were measured using the Bio-Rad Imaging Densitometer (GS-800, Bio-Rad). Band intensities were expressed as absolute values in arbitrary units. The optical density (OD) of each band was plotted against the OD value derived from the Coomassie blue gel.

Gelatine zymography. SDS-PAGE gels $(7.5 \%)$ were prepared with $0.1 \%$ gelatin incorporated during polymerization. In all, $50 \mu \mathrm{g}$ of proteins was loaded on gel lanes and finally subjected to electrophoresis on ice at $40 \mathrm{~mA}$ constant in non-denaturing conditions. After electrophoresis, SDS was removed from gels by washing twice with a renaturating buffer $(50 \mathrm{mM}$ Tris- $\mathrm{HCl}, \mathrm{pH} 7.4$, and $2.5 \%$ Triton $\mathrm{X}$-100) for $20 \mathrm{~min}$ each. Gels were then incubated overnight at $37^{\circ} \mathrm{C}$ in an activation buffer ( $50 \mathrm{mM}$ Tris- $\mathrm{HCl}, \mathrm{pH} 7.4,120 \mathrm{mM} \mathrm{NaCl}, 5 \mathrm{mM} \mathrm{CaCl}$ and $0.04 \% \mathrm{NaN}_{3}$ ). Following the incubation, gels were stained for $30 \mathrm{~min}$ with $0.1 \%$ Coomassie blue (dissolved with $40 \%$ methanol and $10 \%$ acetic acid) and destained in the same solution without Coomassie until clear proteolytic bands appeared on the contrasting blue background. Bands were quantified by densitometry using $\mathrm{NIH}$ - Image J 1.61.

Statistics. For western blotting experiments, immunohistochemistry and novel object recognition test, statistical differences were determined with the Student's $t$-test for unpaired data. For analysis of the MWM latencies and BN-PAGE experiments, the homogeneity of the variances was determined by the Bartlett test (90\% confidence level) and a one-way ANOVA was performed followed by a post-hoc Bonferroni's correction. All data are expressed as mean \pm S.E.M. Analysis of the MWM crosses number employed the non-parametric Kruskal-Wallis test and the threshold for statistically significant differences was set at $P<0.05$.

\section{Conflict of interest}

The authors declare no conflict of interest.

Acknowledgements. We are in debt to Valerio Frazzini and Mary Evangeline Oberschlake for the critical revision and editing of the manuscript. SLS is supported by funds from the Italian Dept. of Education (FIRB 2003; PRIN 2006; PRIN 2008). 
1. Zatta $P$, Drago D, Bolognin S, Sensi SL. Alzheimer's disease, metal ions and metal homeostatic therapy. Trends Pharmacol Sci 2009; 30: 346-355.

2. Sensi SL, Paoletti P, Bush Al, Sekler I. Zinc in the physiology and pathology of the CNS Nat Rev Neurosci 2009; 10: 780-791.

3. Boom A, Authelet M, Dedecker R, Frederick C, Van Heurck R, Daubie V et al. Bimodal modulation of tau protein phosphorylation and conformation by extracellular $\mathrm{Zn2}+$ in human-tau transfected cells. Biochim Biophys Acta 2009; 1793: 1058-1067.

4. Mo ZY, Zhu YZ, Zhu HL, Fan JB, Chen J, Liang Y. Low micromolar zinc accelerates the fibrillization of human tau via bridging of Cys-291 and Cys-322. J Biol Chem 2009; 284: 34648-34657

5. Arispe N, Pollard HB, Rojas E. Zn2+ interaction with Alzheimer amyloid beta protein calcium channels. Proc Natl Acad Sci USA 1996; 93: 1710-1715.

6. Cuajungco MP, Faget KY. Zinc takes the center stage: its paradoxical role in Alzheimer's disease. Brain Res Brain Res Rev 2003; 41: 44-56.

7. Dineley KE, Richards LL, Votyakova TV, Reynolds IJ. Zinc causes loss of membrane potential and elevates reactive oxygen species in rat brain mitochondria. Mitochondrion 2005; 5: 55-65.

8. Sensi SL, Yin HZ, Weiss JH. AMPA/kainate receptor-triggered Zn2+ entry into cortical neurons induces mitochondrial $\mathrm{Zn} 2+$ uptake and persistent mitochondrial dysfunction. Eur J Neurosci 2000; 12: 3813-3818.

9. Rhein V, Song X, Wiesner A, Ittner LM, Baysang G, Meier F et al. Amyloid-beta and tau synergistically impair the oxidative phosphorylation system in triple transgenic Alzheimer's disease mice. Proc Natl Acad Sci USA 2009; 106: 20057-20062.

10. Sensi SL, Rapposelli IG, Frazzini V, Mascetra N. Altered oxidant-mediated intraneurona zinc mobilization in a triple transgenic mouse model of Alzheimer's disease. Exp Gerontol 2008; 43: 488-492

11. Bush Al, Tanzi RE. Therapeutics for Alzheimer's disease based on the metal hypothesis. Neurotherapeutics 2008; 5: 421-432.

12. Adlard PA, Cherny RA, Finkelstein DI, Gautier E, Robb E, Cortes M et al. Rapid restoration of cognition in Alzheimer's transgenic mice with 8-hydroxy quinoline analogs is associated with decreased interstitial Abeta. Neuron 2008; 59: 43-55.

13. Cherny RA, Atwood CS, Xilinas ME, Gray DN, Jones WD, McLean CA et al. Treatment with a copper-zinc chelator markedly and rapidly inhibits beta-amyloid accumulation in Alzheimer's disease transgenic mice. Neuron 2001; 30: 665-676.

14. Linkous DH, Adlard PA, Wanschura PB, Conko KM, Flinn JM. The Effects of enhanced zinc on spatial memory and plaque formation in transgenic mice. J Alzheimers Dis 2009; 18: 565-579.

15. Stoltenberg M, Bush Al, Bach G, Smidt K, Larsen A, Rungby J et al. Amyloid plaques arise from zinc-enriched cortical layers in APP/PS1 transgenic mice and are paradoxically enlarged with dietary zinc deficiency. Neuroscience 2007; 150: 357-369.

16. Adlard PA, Parncutt JM, Finkelstein DI, Bush Al. Cognitive loss in zinc transporter-3 knockout mice: a phenocopy for the synaptic and memory deficits of Alzheimer's disease? $J$ Neurosci 2010; 30: 1631-1636.

17. Xu H, Gao HL, Zheng W, Xin N, Chi ZH, Bai SL et al. Lactational zinc deficiency-induced hippocampal neuronal apoptosis by a BDNF-independent TrkB signaling pathway. Hippocampus 2010.

18. Connor B, Young D, Yan Q, Faull RL, Synek B, Dragunow M. Brain-derived neurotrophic factor is reduced in Alzheimer's disease. Brain Res Mol Brain Res 1997; 49: 71-81.

19. Peng S, Wuu J, Mufson EJ, Fahnestock M. Precursor form of brain-derived neurotrophic factor and mature brain-derived neurotrophic factor are decreased in the pre-clinical stages of Alzheimer's disease. J Neurochem 2005; 93: 1412-1421.

20. Hwang JJ, Park MH, Choi SY, Koh JY. Activation of the Trk signaling pathway by extracellular zinc. Role of metalloproteinases. J Biol Chem 2005; 280: 11995-12001.

21. Billings LM, Oddo S, Green KN, McGaugh JL, LaFerla FM. Intraneuronal Abeta causes the onset of early Alzheimer's disease-related cognitive deficits in transgenic mice. Neuron 2005; 45: 675-688.

22. Brown MW, Aggleton JP. Recognition memory: what are the roles of the perirhinal cortex and hippocampus? Nat Rev Neurosci 2001; 2: 51-61.

23. Buffalo EA, Reber PJ, Squire LR. The human perirhinal cortex and recognition memory. Hippocampus 1998; 8: 330-339.

24. Winters BD, Forwood SE, Cowell RA, Saksida LM, Bussey TJ. Double dissociation between the effects of peri-postrhinal cortex and hippocampal lesions on tests of object recognition and spatial memory: heterogeneity of function within the temporal lobe. J Neurosci 2004; 24: 5901-5908

25. Oddo S, Caccamo A, Kitazawa M, Tseng BP, LaFerla FM. Amyloid deposition precedes tangle formation in a triple transgenic model of Alzheimer's disease. Neurobiol Aging 2003 24: 1063-1070.

26. Yao J, Irwin RW, Zhao L, Nilsen J, Hamilton RT, Brinton RD. Mitochondrial bioenergetic deficit precedes Alzheimer's pathology in female mouse model of Alzheimer's disease. Proc Natl Acad Sci USA 2009; 106: 14670-14675.

27. Prasad AS. Zinc deficiency: its characterization and treatment. Met lons Biol Syst 2004; 41 103-137.

28. Maret W, Sandstead $\mathrm{HH}$. Zinc requirements and the risks and benefits of zinc supplementation. J Trace Elem Med Biol 2006; 20: 3-18.

29. Wuehler SE, Peerson JM, Brown KH. Use of national food balance data to estimate the adequacy of zinc in national food supplies: methodology and regional estimates. Public Health Nutr 2005; 8: 812-819.

30. Maynard CJ, Cappai R, Volitakis I, Laughton KM, Masters CL, Bush Al et al. Chronic exposure to high levels of zinc or copper has little effect on brain metal homeostasis or Abeta accumulation in transgenic APP-C100 mice. Cell Mol Neurobiol 2009; 29: 757-767.

31. Hirata-Fukae C, Li HF, Hoe HS, Gray AJ, Minami SS, Hamada K et al. Females exhibit more extensive amyloid, but not tau, pathology in an Alzheimer transgenic model. Brain Res 2008; 1216: 92-103.

32. Lee JY, Kim JH, Hong SH, Lee JY, Cherny RA, Bush Al et al. Estrogen decreases zinc transporter 3 expression and synaptic vesicle zinc levels in mouse brain. J Biol Chem 2004; 279: 8602-8607.

33. LaFerla FM, Green KN, Oddo S. Intracellular amyloid-beta in Alzheimer's disease. Nat Rev Neurosci 2007; 8: 499-509.

34. Oddo S, Caccamo A, Shepherd JD, Murphy MP, Golde TE, Kayed R et al. Tripletransgenic model of Alzheimer's disease with plaques and tangles: intracellular Abeta and synaptic dysfunction. Neuron 2003; 39: 409-421.

35. Kang H, Schuman EM. Long-lasting neurotrophin-induced enhancement of synaptic transmission in the adult hippocampus. Science 1995; 267: 1658-1662.

36. Zuccato $\mathrm{C}$, Cattaneo $\mathrm{E}$. Brain-derived neurotrophic factor in neurodegenerative diseases. Nat Rev Neurol 2009; 5: 311-322.

37. Huang $Y Z$, Pan E, Xiong ZQ, McNamara JO Zinc-mediated transactivation of TrkB potentiates the hippocampal mossy fiber-CA3 pyramid synapse. Neuron 2008; 57 546-558.

38. Besser L, Chorin E, Sekler I, Silverman WF, Atkin S, Russell JT et al. Synaptically released zinc triggers metabotropic signaling via a zinc-sensing receptor in the hippocampus. J Neurosci 2009; 29: 2890-2901.

39. Backstrom JR, Lim GP, Cullen MJ, Tokes ZA. Matrix metalloproteinase-9 (MMP-9) is synthesized in neurons of the human hippocampus and is capable of degrading the amyloid-beta peptide (1-40). J Neurosci 1996; 16: 7910-7919.

40. White AR, Du T, Laughton KM, Volitakis I, Sharples RA, Xilinas ME et al. Degradation of the Alzheimer disease amyloid beta-peptide by metal-dependent up-regulation of metalloprotease activity. J Biol Chem 2006; 281: 17670-17680.

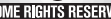

Cell Death and Disease is an open-access journal published by Nature Publishing Group. This work is licensed under the Creative Commons Attribution-Noncommercial-No Derivative Works 3.0 Unported License. To view a copy of this license, visit http://creativecommons.org/licenses/by-nc-nd/3.0/

Supplementary Information accompanies the paper on Cell Death and Disease website (http://www.nature.com/cddis) 